2021-08-08

\title{
Exploring the applicability of the socially assistive robot Stevie in a day center for people with dementia*
}

\section{Taylor, L}

http://hdl.handle.net/10026.1/17945

\subsection{9/ro-man50785.2021.9515423}

2021 30th IEEE International Conference on Robot \& Human Interactive Communication (RO-MAN)

IEEE

All content in PEARL is protected by copyright law. Author manuscripts are made available in accordance with publisher policies. Please cite only the published version using the details provided on the item record or document. In the absence of an open licence (e.g. Creative Commons), permissions for further reuse of content should be sought from the publisher or author. 


\title{
Exploring the applicability of the socially assistive robot Stevie in a day center for people with dementia*
}

\author{
Lloyd Taylor, Angela Downing, Gabriel Aguiar Noury, Giovanni Masala, Marco Palomino, Conor \\ McGinn, and Ray Jones
}

\begin{abstract}
Socially assistive robots have the potential to help keep people with dementia cognitively active and entertained. This is important for their wellbeing. We explored staff perceptions of the acceptability of a new humanoid robot, Stevie, in an adult day care center for people with dementia. Stevie was deployed over 2 weeks in the day center to entertain 40 guests with dementia with three activities: (i) musical bingo, (ii) quizzes, and (iii) meet and greet. Nine staff members were asked what went well, whether the robot operated as expected, experiences of any technical issues, and the extent to which it hindered or aided their duties. Staff also rated guest engagement, guest enjoyment, and whether they were able to spend more time with guests. The sessions were successfully delivered. Staff reported that Stevie operated as expected, guest engagement with the robot was high, interactions with the robot were natural and the robot's novelty helped engagement. Use of the robot gave staff more time to attend to guests' needs. Suggested improvements included improvements to the robot's voice and adding a pause function during activities. With greater autonomy and/or extended use of its telepresence functionality, use of Stevie could become cost effective in such settings.
\end{abstract}

\section{INTRODUCTION}

The aging population creates increased demand for elderly care that is leading to a chronic shortage of care staff [1]. Staff shortages cause a vicious cycle of burnout, work-related stress and further absence or abandonment of the profession [2]. For older people in need of support services, staff shortage translates into a reduction of personalized care, and an increase of social isolation and loneliness [3].

As the state of the art in automation advances, more complex social needs can be addressed using robots [4]. Robotic and autonomous systems serving different applications in the health and social care sector are primed to become a key technology. Socially assistive robots (SARs) robots that help humans through social interaction [5] - could be used in new roles to assist staff and meet health and social care challenges [6]. SARs can be categorized into two groups: service robots and companion robots. Companion robots are generally associated with increasing the psychological status and overall well-being of users. For example, studies since 2000 have shown the most well-known companion robot, Paro the seal [7] reduces loneliness among older people [8], and can improve mood and anxiety among older people [9].

*This research has been partly supported by the eHealth Productivity and Innovation in Cornwall and the Isles of Scilly project partly funded by the ERDF (project number [05R18P02814] and the Interreg 2 Seas Mers Zeeën AGE'IN project (2S05-014)

Lloyd Taylor, Ray Jones, Gabriel Aguiar, Noury and Marco Palomino worked for University of Plymouth; Giovanni Masala worked for Manchester
In contrast, service robots can aid activities of daily living and have shown potential supporting carers. For instance, Law and colleagues [10] assessed the usefulness of the Silbot humanoid assistive robot in a care setting, from both care receivers and experts in aged care perspectives and concluded that there was potential to reduce caregiver burden. However, despite much of the promises, the adoption of this technology in elderly care is not completely clear. There has been a lack of research in group settings [11]. To address this gap, this paper explores the use of a humanoid robot in the communal setting of a day center for people with dementia, deployed and used daily by the center staff for ten working days over a period of two weeks.

Poberznik et al [12] investigated experiences of the Pepper robot in a sample of older patients with schizophrenia and/or dementia in a rehabilitation center in Japan. They reported that additional control buttons could ease use. Further, a study by Cormons et al. [13] testing social robot acceptance using a panel of students who reported that the lack of feedback from the robot inhibited them from adapting their own behavior to that of the robot.

Stevie [14] is a new humanoid socially assistive robot developed specifically to be used in an eldercare setting. Stevie has been designed addressing issues raised by these studies. Firstly, the control system gives the user full control over the robot including the ability to control volume levels of the robot's speech and media as well as motions and movements. Secondly, Stevie has a range of facial expressions designed to convey unambiguous emotions. Prior to this study, Stevie had only been evaluated in the US [17]; this study was the first trial of Stevie in the United Kingdom (UK). Importantly, in the current study, Stevie was tested at a day center for older adults living with dementia, unlike the US deployment where users were receiving support for a continuum of other age-related care needs.

Qualitative methods were used to explore Stevie's interactions with guests at the day center. Specifically, we were interested in how well Stevie interacted with guests of the center, whether the robot had operated as expected, experiences of any technical issues, and the extent to which Stevie's presence and activities hindered or aided staff's duties. As well as these free text responses, we asked staff to rate how well guests interacted with Stevie, whether guests enjoyed

Metropolitan University, and Conor McGinn worked for Trinity College Dublin. Angela Downing is Owner and Manager of the Reflections Day Center in Camborne, Cornwall, hosted the study and supervised the care staff and day center guests. Conor McGinn is the co-inventor of the Stevie Robot providing technical support in the set up of the study but was not involved in the operation or data collection for the evaluation. 
Stevie's activities, and whether Stevie's presence enabled staff to spend more time with guests.

\section{AIMS AND DESIGN}

\section{A. Aims}

We hypothesized that the robot would be positively perceived by the day center staff and guests. We explored the benefit of the robot's constant presence at the day center across three dimensions; engagement, enjoyment, and staff time spent with guests. We expected there to be a high level of engagement and enjoyment as a response to staff and guests' interactions with Stevie, and that Stevie's presence and activities at the day center would allow staff to allocate higher attention to guests, and spend more time with them.

\section{B. Design}

A mixed-methods approach was used to explore staff views, experiences and perceptions of Stevie. This study was reviewed and approved by the Faculty of Health Research Ethics Committee (Ref: 19/20-1193; dated 18/11/19). Stevie was deployed to run activities for the guests of the center. These activities were musical bingo, an interactive quiz and meet and greet sessions over 10 working days. Questionnaires given to the staff participants and interviews were conducted within two days of the session being run.

\section{Setting AND PARTicipants}

The Reflections Day Centre located in Camborne, Cornwall, UK is a day center specializing in the care of people with dementia. Cornwall is a rural county at the western periphery of the UK, one of the poorer regions in Europe with an older than average population. It has an ageing population well above the national average ( $24 \%$ over 65 compared to $18 \%$ for the UK in 2015). Reflections Day Centre operates during the day providing group and individual activities. Guests at the day center arrive between 9:30-10:00am and stay until $3: 30 \mathrm{pm}$. Guests attending are usually in groups of 6-8 people in a room with an average of eighteen people attending each day. Most guests who attend the center live at home with their spouses and families and a small percentage live at home alone. Typical activities provided at Reflections include reminiscence therapies, cognitive stimulation therapies, arts and crafts, music, singing, dance and movement, quizzes and games.

The study sample comprised of nine members of staff who were either full time, part-time, or voluntary workers at Reflections. The primary duties of our participants were to provide day care services to the guests - people with dementia.

\section{The Stevie Robot}

The robot used in the study was a humanoid robot known as Stevie [14] (Figure 1). Stevie, as deployed, was in its second design iteration following research by the development team at Trinity College Dublin, Ireland.

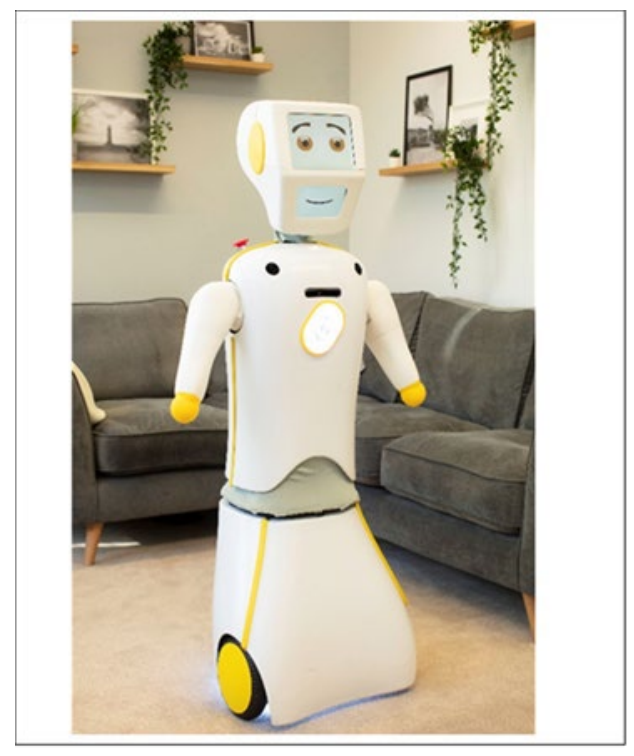

Figure 1. The Stevie Robot.

Stevie comprises a wheeled differential-drive mobile base with degrees of freedom in its waist (pitch, yaw), shoulders (pitch, yaw) and neck (roll, yaw, pitch). It has two on-board computers (Intel NUC) and possesses a range of sensors including a 2D LIDAR (Hokoyu URG), RGBD camera (Intel RealSense D430) and four on-board cameras.

Two screens on the head of the robot make up its face with the upper screen displaying its eyes and the lower screen displaying its mouth. The robot can speak via preprogramed scripts or through a text-to-speech system run on a laptop wirelessly connected to the robot. All tele-operation was performed by a member of the research team who was physically present at the facility. Remote teleoperation may have been possible (i.e. using information from the cameras situated around the robot including: two front-facing cameras near the shoulders, a rear facing camera on its back and a popup camera in its head), however, this feature was not trialed in this study.

Design features such as Stevie's ability to convey facial expressions were developed with the aim of promoting unambiguous interactions between a human and the robot. Further, Stevie's arms are purposively designed so that it is apparent that they are non-functioning limbs. The Pepper platform has very human like hands and arms for the purpose of gesturing during conversation. However, these human-like limbs can lead people into assuming that the robot is capable of carrying out complex motor functions beyond gesturing. The clearly identifiable facial expressions and obviously limited functionality of the robot's limbs are designed to give intuitive interactions and set expectancies of the capabilities from introduction. Moreover, the Stevie platform has a user interface via a controller laptop that allows a user to take partial or full control of the system if the need arises. Conversely, Pepper can be controlled via the Choregraphe suite, however, this UI is designed to upload programs to the robot and is not a remote-controlled system. 


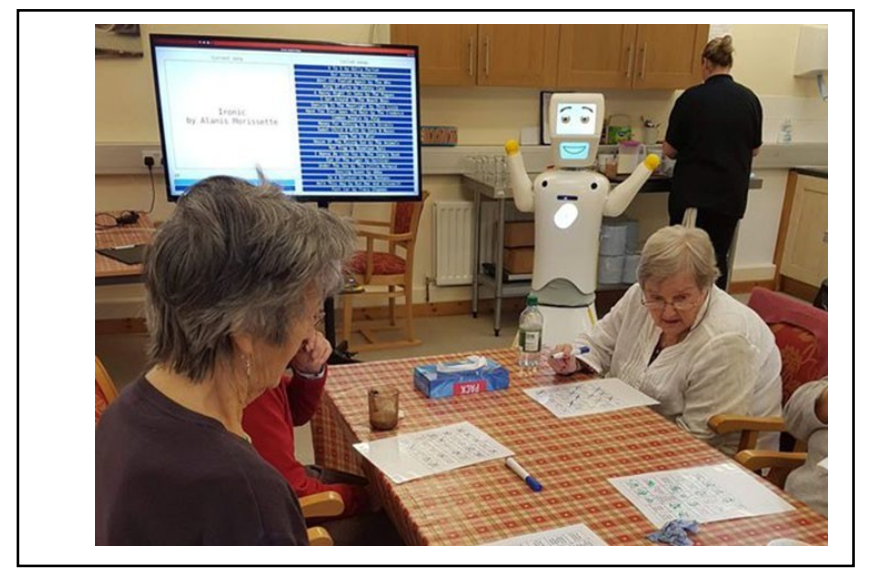

Figure 2. Stevie running the musical bingo session. Stevie verbally called the songs while their titles were displayed visually on the TV.

Stevie has a range of operational functions such as, but not limited to, human detection, face detection, autonomous environment navigation, speech and speech recognition which are each implemented through the Robot Operating System (ROS).

Further, unlike many similar robots, a user can take full control of Stevie viaa bespoke desktop application, thus allowing users to deploy the robot in a diverse range of situations. This control mechanism is in the form of a graphical user interface (GUI) on a laptop which gives the operator full control of the robot's navigation, limbs, cameras, facial expressions, speech recognition and pre-programmed movement sequences such as the dancing motion.

Moreover, Stevie can stream information to a monitor or TV enabling the robot to play host to a range of interactive activities that would usually be conducted by a human caregiver.

\section{A. Level of autonomy}

Certain tasks were tele-operated by the researcher or a staff member such as navigating the robot through the center and the meet and greet activities where close proximity with guests was anticipated. However, during the quiz and bingo activities, the only input from the user was to start the activity. Therefore, once the activity was started, it is reasonable to assume that the robot could have been left to carry out the activity without monitoring by a member of staff. In our study, the researcher was in the same room as the robot.

Stevie also has an RGBD camera and LIDAR that detects potential collisions and stops the robot from moving if an obstacle is detected. The arms of the robot have also been designed in such a way that they will give way if they come into contact with an obstacle.

\section{B. Bespoke Activity Applications}

Two activity sessions per day were carried out using the robot, with each session lasting between 45-60 minutes. Morning sessions comprised of either a quiz or bingo, with all afternoon sessions being open-ended "meet and greet" sessions with the robot. Twenty sessions were run across ten days: 5 musical bingo, 10 meet and greet, and 5 quizzes.

Musical bingo: This application implemented the same premise as bingo, with numbers being replaced with clips of well-known songs. Songs were selected based on their popularity in the UK and tailored to the age of the guests at the center. Each player was given a bingo card and a marker (Figure 2). Music clips were played through the robot's speakers while the name of the song was displayed on the GUI being cast on to a large screen TV from the laptop. The current song was displayed prominently before moving over to a list of played songs.

Interactive quiz: A multiple choice general knowledge quiz tailored to the age and location (Cornwall) of the guests of the center was developed. Using a similar GUI as the bingo, up to four teams were able to play the quiz. Each team chose a team name that was manually entered into the program by a member of staff. Once the game started, the team names and their corresponding score was displayed prominently on the screen. Questions were announced verbally by the robot, followed by four multiple choice answers. The questions and possible answers were also shown on the accompanying TV monitor. Each team had a wireless PS3 Buzz controller [20] which was used to enter answers to the multiple-choice questions. Upon selecting one of the four responses on the controller, the corresponding team name would be highlighted to confirm they had given an answer. Once all four teams had submitted an answer, their choice was displayed on the screen while the robot called the correct answer and named the team or teams that had answered correctly. Team scores were updated and displayed next to the team names accordingly.

"Meet and Greet": "Meet and greet" sessions were less formal activities carried out using the Stevie robot. Although the Stevie system is capable of navigating its environment and has speech recognition capabilities, the "meet and greet" sessions were mostly controlled by either the experimenter or the care staff using the robots GUI controller. During the "meet and greet" session, the robot would be guided into one of the activity rooms. Guests would be seated around the edge of the room with the robot in the middle. The robot was steered to individuals and would ask pre-programmed questions, or the text-to-speech system would be employed to personalize the conversation. During the "meet and greet", the robot would also make use of its library of jokes. The robot had some preloaded music which it would play and dance to. This was used to encourage the guests of the center to dance as a way of promoting interaction with the robot (Figure 3). 


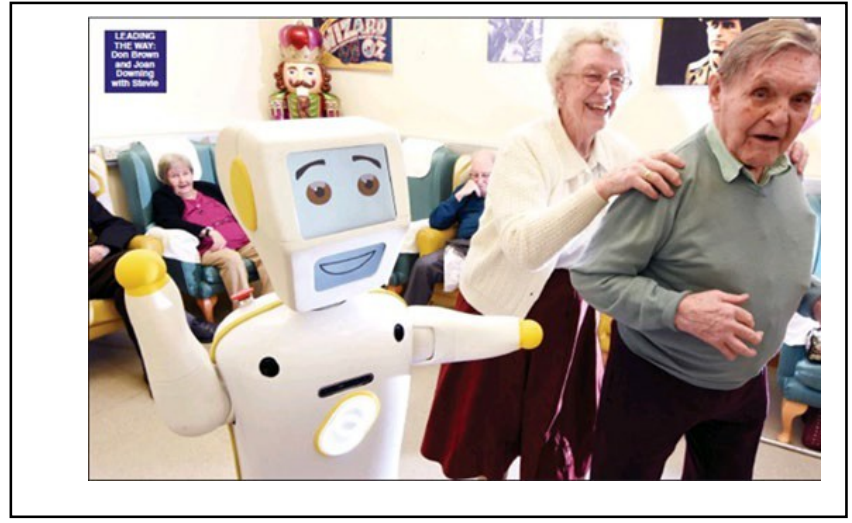

Figure 3. Guests dancing inspired by Stevie

\section{DAta Collection}

Data were collected from questionnaires, interviews and a researcher diary. Quantitative data were collected using questionnaires to gain general staff perception of guest engagement, guest enjoyment, and their time spent with guests. At the end of each session, three statements were given to staff participants: 1) Session guests were engaged in the session (engagement). 2) Session guests enjoyed the session (enjoyment). 3) I was able to spend more time with the seniors (time spent with guests). Staff responded on a five-point

Likert scale: strongly disagree, disagree, neutral, agree, strongly agree. This questionnaire gave an overall assessment.

To gain a deeper understanding into Stevie's impact at the center, we carried out semi-structured interviews, one member of staff at the end of each session, to explore staff's views, perceptions, and experiences of the session with Stevie. Staff were asked about what went well, whether the robot operated as expected, experiences of any technical issues, and the extent to which it hindered or aided their duties.

Finally, a researcher diary was kept to note issues with the robot or other episodes or influences on its use with guests or staff perceptions.

\section{V1. DATA ANALYSIS}

Questionnaire data were used for simple frequency of response. Eight of the nine staff members were interviewed. Interview data were transcribed verbatim and thematically analyzed guided by Braun and Clarke's [15] six-step guide. These steps are: familiarizing yourself with data by reading (and re-reading) the transcript, developing codes by systematically generating phrases to segment qualitative data and capture important concepts, searching for themes which describe common ideas or conceptualizations derived from codes, reviewing themes, defining themes, naming themes, and reporting. Thematic analysis is a flexible and appropriate method of summarizing qualitative data in the form of transcribed text or open-ended responses [15].

\section{V11 RESULTS}

Thirty-eight questionnaires were collected from nine members of staff after ten sessions. All members of staff for all sessions, agreed or strongly agreed that guests were engaged in the session, enjoyed the session, and that staff were able to spend more time with guests. There was no difference between the three types of activity.

The thematic analysis of interviews was very consistent with these overall assessments.

\section{A. What went well}

There were three sub-themes to what went well: Guest engagement, interactions, and novelty. Staff expressed how Stevie encouraged guest engagement during the robot's activities as Stevie re-directed attention and focus. For example: "Yes, yeah it made it completely different from what we normally do. And he actually made quite a few people like the lady that won't normally dance get up and dance" and "Stevie was a good focus point. He comes in, you know. Clients were tired. He comes into the room and everyone is looking at him, interested. Like I say good focus point. He did really help yesterday really helpful".

Staff cited cases where the guests showed behaviors indicating that they had anthropomorphized Stevie. This became evident in the guests' two-way interactions with Stevie, for example: "And I think that was quite nice that they kind of spoke to him as he was like a normal person almost" and "It was good that it responded and that the humor went well. I think people kind of find it as turning into a robot as human because it is a human thing".

Participants pointed out the novelty Stevie brought, for example: "They were really interested. It was something new" and "It's almost because he is such a novelty at the moment they are a bit star struck they sort of stare and don't know what to do".

On the second day of the study there was some disruption at the center with one guest wanting to leave. The guest had become quite upset but as Stevie was coming from a session the guest engaged with the robot and the researcher. The guest became very interested in what Stevie did and why he was there. The presence of the robot and the subsequent conversation between the guest, researcher and Stevie (the researcher activated pre-programmed question and responses from the robot's console) distracted the guest from their attempts to leave the center. During the conversation guests that had been in the Stevie-led activity started to move past on their way to lunch. This gave the previously distressed guest other avenues of conversation regarding the robot. At the suggestion of a member of staff the guest went with the group to go and have lunch. (Diary data).

\section{$B$. Whether the robot operated as expected}

There were four sub themes to whether the robot operated as expected: useful functions, Stevie's features, individual differences, and inclusivity. Staff discussed Stevie's useful 
functions while the robot interacted with guests, for example: "I was pretty happy with all the bodily expressions he was doing as well [laughs] raising his arms and everything" and "it was a case of you look at the TV you saw the colors you saw the answers within the colors and all I had to do was show the clients the remote that I had and all I had to do was point to a color and say is it this one, what do you think?".

Staff also noted guests experiencing instances of anthropomorphism embedded in Stevie's features, for example: "I think his different facial expression that he done went really well because they could all they could all relate to it they knew what each expression was". Some participants noted that guests' interactions with Stevie were contingent upon individual differences in guests, for example: "I think a couple of more of them found it a bit more difficult to understand what he was saying a couple of times we had a couple of ladies that were a bit hard of hearing" and "For some of them it might have been a bit difficult. But because they are so varied in their cognitive impairment that would be difficult to manage for Stevie".

In several cases, staff expressed that some entertainment components Stevie offered could have supported inclusivity, for example: "I had one guest in there that was in his 60's and one in his 90's so some music that would have suited that era as well, that generation" and "And maybe the song choice, one gent knew most of them and absolutely loved it but the others were sort of like ninety odd and were like what the hell is Bon Jovi [laughs]".

\section{Technical issues}

Staff pointed out some technical issues of Stevie that could be translated into future improvements of the way the robot operates. For example, one said "I think being able to pause it so we could play the songs for longer would have been good". Others commented on the voice; "And sometimes the voice went a bit, it was hard to understand what it was saying because of the robotic voice". The robot's shoulders have pitch and roll dimensions of movement giving it the ability to convey more natural humanoid body language. A design feature of the shoulders is that they will fail before being able to apply enough force to cause injury. During one of the sessions a guest held the robot's limb while dancing with the robot. This resulted in the desired failure of the limb. Maintenance was required to restore it to working order for the next day's session (Diary data).

\section{Hindered or aided staff's duties}

There were three sub-themes to whether Stevie hindered or aided staff's duties: Staff enablement, individual differences, groups. One prominent comment throughout was the observation that Stevie's activities allowed participants (staff) to attend to other duties, interact more with guests, and provide more support to guests, thus supporting staff enablement, for example: "So with Stevie keeping the guests, you know, stimulated and occupied it meant one of us was able to go off, make the drinks and still have two people, like three members of staff in the room" and "And it gave us the opportunity then do go to someone one to one and ask them what they thought. Instead of having to talk to the whole group, you could do it more one to one" and "Gave us more time with the clients so obviously we could just do the bingo and gave us time to actually be with them and interact with them more".

\section{V111 DISCUSSION}

This study explored how staff at Reflections Day care center for people with dementia perceived Stevie, a social robot set out to provide a range of activities to the center's guests.

The nine participants, members of staff, agreed that guests were engaged with the robot, enjoyed it, and that this allowed them to spend more time with guests. This was consistent across the three activities (musical bingo, meet and greet, interactive quiz).

The quantitative ratings were supported by staff views in interviews. They reported that guests were engaged and interacted with Stevie in a positive way during its activities. Their interactions seemed natural, as it was evident that they engaged with Stevie as they would with another person, which facilitated interactions. There is research to indicate that such stimulation from social interaction in people with dementia can benefit well-being and quality of life [16].

Stevie's functions operated as expected during its time at the center, with its facial expressions and bodily movements accompanying the well-received activities, which Stevie offered to guests. Some staff highlighted some areas for further improvement for instance, addressing individual differences within guests such as age, cognitive impairment, and hearing. Further improvements were suggested such as ability to pause songs, or improving the quality of Stevie's voice. Varying the complexity of activities could be a way forward. Another avenue may be to re-iterate instructions, give reminders, or offer simpler activities (e.g. easier questions on the quiz) if Stevie registers a number of wrong responses. Stevie could also be refined to be more bespoke depending on the context and population it is expected to serve.

Importantly, our study showed that Stevie aided staff by allowing them to attend to duties, interact more with guests, and provide more support while Stevie was present and carried out activities. Arguably, this can contribute to more tailored attention and support of staff to guests and potentially increase guest satisfaction. Although during the study Stevie was closely monitored by a researcher or center staff via its control computer during each session, the bingo and quiz activities required little to no input from a user. Further, Stevie is already equipped with hardware and design features that will allow the system to be ever more autonomous as its software is developed. In the future Stevie has the potential to operate fully autonomously.

One notable difference between the Stevie platform and robots such as Pepper is that Stevie is able to cast interactive media to third party screens as well have hardware such as 
quiz controllers wirelessly connected for greater user interfacing. This allows for a more inclusive and wider ranging set of interactions, as casting to a large screen allowed all guests the ability to view the media rather than just those close to the robot as would be the case with the Pepper platform. Further, the use of the quiz controllers allowed guests the ability to take part in the activity ensuring their input was confined to the context of the activity.

Since the current study lacked a comparison group, future research could focus on implementing a control group to allow for comparisons. Specifically, future studies can use mixed methods to compare Stevie to other humanoid robots. This could provide an insight into how Stevie's design and functions operate compared to other systems in the elderly care setting.

A further line of research could implement more standardized measures designed for measuring dimensions of human-robot interactions. Robinson et al. [17] developed psychometric tools to assess incentives, intentions, and selfefficacy to use social robots. Based on data obtained from such measures, Stevie's features and characteristics can be refined to reflect such dimensions as they may predict acceptability and use of Stevie [18].

Another interesting point of focus could be to test whether people with dementia show improved cognitive abilities or a cease in decline of such abilities following interactions with Stevie. This would be beneficial to the way centers like Reflections operate as it could potentially impact guests' quality of life and well-being [16]. It may enable care workers to provide more focused, one-to-one support to guests, which is what we showed in this study.

In conclusion, this initial study into implementing a social robot, Stevie, into a day center for people with dementia showed promising results and provided insight into the way Stevie could benefit the way of living in such contexts. As predicted, the presence of Stevie empowered staff to allocate their attention to guests and spend more time with them. Going forward there is much potential for future research to refine the way Stevie, and other social robots, operate in these settings to allow for increased benefits.

\section{REFERENCES}

[1] All-Party Parliamentary Group on Global Health 2016. [online] Available at: <http://www.appg-globalhealth.org.uk/> [Accessed 20 January 2021].

[2] Duffy, B., Oyebode, J. R., \& Allen, J. (2009). Burnout among care staff for older adults with dementia: The role of reciprocity, selfefficacy and organizational factors. Dementia, 8(4), 515-541.

[3] Neves BB, Sanders A, Kokanović R. "It's the worst bloody feeling in the world": Experiences of loneliness and social isolation among older people living in care homes. Journal of Aging Studies. 2019 Jun $1 ; 49: 74-84$

[4] Dahl TS, Boulos MN. Robots in health and social care: A complementary technology to home care and telehealthcare? Robotics. 2014 Mar;3(1):1-21.
[5] Bedaf S, Gelderblom GJ, De Witte L. Overview and categorization of robots supporting independent living of elderly people: what activities do they support and how far have they developed. Assistive Technology. 2015 Apr 3;27(2):88-100.

[6] Prescott, T.J. and Caleb-Solly, P., 2017. Robotics in social care: a connected care EcoSystem for independent living. https://www.ukras.org/wpcontent/uploads/2018/10/UK_RAS_wp_social_spread_low_res_ref.pd $\mathrm{f}$

[7] Parorobots.com. 2014. PARO Therapeutic Robot. [online] Available at: $<$ http://www.parorobots.com/ $>$ [Accessed 20 January 2021].

[8] Robinson, H., MacDonald, B., Kerse, N., \& Broadbent, E. (2013). The psychosocial effects of a companion robot: a randomized controlled trial. Journal of the American Medical Directors Association, 14(9), 661-667.

[9] Moyle, W., Cooke, M., Beattie, E., Jones, C., Klein, B., Cook, G., \& Gray, C. (2013). Exploring the effect of companion robots on emotional expression in older adults with dementia: a pilot randomized controlled trial. Journal of gerontological nursing, 39(5), 46-53.

[10] Law, M., Sutherland, C., Ahn, H. S., MacDonald, B. A., Peri, K., Johanson, D. L., ... \& Broadbent, E. (2019). Developing assistive robots for people with mild cognitive impairment and mild dementia: a qualitative study with older adults and experts in aged care. BMJ open, 9(9), e031937.

[11] IFR, 2016. World Robotics Report 2016 [WWW Document]. URL https://ifr.org/ifr-press-releases/news/world-robotics-report-2016 (accessed 9.9.17)

[12] McGinn, C., Bourke, E., Murtagh, A., Donovan, C., Lynch, P., Cullinan, M. F., \& Kelly, K. (2020). Meet Stevie: a Socially Assistive Robot Developed Through Application of a 'DesignThinking'Approach. Journal of Intelligent \& Robotic Systems, 98(1), 39-58.

[13] Poberznik A, Merilampi S. 13 Older adults' experiences with Pepper humanoid robot. Tutkimusfoorumi.:148.

[14] Cormons L, Poulet C, Pellier D, Pesty S, Fiorino H. Testing social robot acceptance: what if you could be assessed for dementia by a robot? A pilot study. In2020 6th International Conference on Mechatronics and Robotics Engineering (ICMRE) 2020 Feb 12 (pp. 92-98). IEEE.

[15] Braun, V., \& Clarke, V. (2006). Using thematic analysis in psychology. Qualitative research in psychology, 3(2), 77-101.

[16] Aguirre, E., Woods, R. T., Spector, A., \& Orrell, M. (2013). Cognitive stimulation for dementia: a systematic review of the evidence of effectiveness from randomised controlled trials. Ageing research reviews, 12(1), 253-262.

[17] McGinn C, Bourke E, Murtagh A, Donovan C, Cullinan MF. Meeting Stevie: perceptions of a socially assistive robot by residents and staff in a long-term care facility. In2019 14th ACM/IEEE International Conference on Human-Robot Interaction (HRI) 2019 Mar 11 (pp. 602-603). IEEE.

[18] Robinson, N. L., Connolly, J., Johnson, G. M., Kim, Y., Hides, L., \& Kavanagh, D. J. (2018). Measures of incentives and confidence in using a social robot. Science Robotics, 3(21), Article-number.

[19] Latikka, R., Turja, T., \& Oksanen, A. (2019). Self-efficacy and acceptance of robots. Computers in Human Behavior, 93, 157-163.

[20] https://www.manua.ls/sony/wireless-buzz-buzzers/manual

\section{ACKNOWLEDGEMENT}

We thank Jen Whitfield and all the staff, guests and their families at Reflections Day Centre, Camborne, Cornwall. In particular, we thank those guests who gave permission for their photos to be included in the paper. This research has been partly supported by the eHealth Productivity and Innovation in Cornwall and the Isles of Scilly project partly funded by the ERDF (project number [05R18P02814] and the Interreg 2 Seas Mers Zeeën AGE'IN project (2S05-014).

\section{CONFLICT OF INTEREST}

Conor Mc Ginn is a co-inventor of Stevie. However, he was not directly involved with the data collection and analysis of this study. 